\title{
Benefits of noise
}

DOI:

10.1038/nrg2057

URLs

Entrez Gene

GAL1

http://www.ncbi.nlm.nih.

gov/entrez/query.fcgi?db=ge

ne\&cmd=Retrieve\&dopt=full_

reportElist_uids $=852308$
Factors such as transcription, translation, chromatin remodelling and subcellular localization contribute to gene expression differences between cells. New work now looks at the consequences of such variability. Using a modified GAL1 promoter in Saccharomyces cerevisiae, Blake et al. show that stability of the transcription complex affects gene expression variability, and that increased variability can be beneficial following a change in environment.

From previous work, the authors knew that sequence variation in the normally highly conserved TATA box can directly affect transcription activation. They therefore designed several TATA-box variants of the wild-type GAL1 promoter, reasoning that doing so would affect the binding of TATA-box binding proteins (TBP) leading to altered levels of transcription (in this system, of yeast EGFP (enhanced green fluorescent protein)). Using flow cytometry to measure transcriptional output, they found that expression from mutant promoters was more variable, indicating that the stability of the transcriptional scaffold, which is built around TBP, can influence phenotypic variability.
Next the authors built a computational model of promoter occupancy under different conditions and at different stages during transcription. Their simulations indicated that cells in which stable transcriptional complexes are formed show abrupt changes in protein levels, resulting in a high degree of variability. Moreover, the simulations showed that the speed at which a cell responds to a stimulus is linked to the stability of a transcriptional scaffold at the responding promoter.

Using an array system that allowed them to simultaneously monitor the fluorescence of many individual cells over time, the authors showed that modulating the sequence of a TATA box does indeed affect the frequency and magnitude of gene expression bursts in individual cells within a population.

Variable responses to a stimulus could have profound effects on a cell's adaptability to a changing environment. To study this, Blake et al. created a system in which two promoter variants that mediate distinct expression responses drive expression of an antibiotic resistance gene. They used cell growth and survival after antibiotic treatment as a readout of promoter 'performance'. The variant that promotes expression bursts, and therefore a rapid response, conferred higher fitness on its carrier. Other experiments showed that variability in response can be beneficial at high levels of stress, but that increased variability can be less advantageous at low levels of stress. The authors therefore propose that TATA-box promoters might have evolved to enable rapid responses from a subset of cells. In fact, a recent genome-wide analysis showed that most TATA-containing promoters are associated with stress-response genes in S. cerevisiae, whereas housekeeping genes are associated with TATA-less promoters. By showing that TATA-containing promoters can facilitate gene expression bursts, thereby directly influencing the level of noise in gene expression, Blake et al. provided mechanistic insights into the differences between the regulation and output of stress-response genes and those that do not respond to stress or environment.

Magdalena Skipper

ORIGINAL RESEARCH PAPER Blake, W. J. et al.

Phenotypic consequences of promoter-mediated transcriptional noise. Mol. Cell 24, 853-865 (2007) FURTHER READING Kaern, M. et al.

Stochasticity in gene expression: from theories to phenotypes. Nature Rev. Genet. 6, 451-464 (2005) 